\title{
Assessing the maturity of collaborative networks: a case study analysis in the Italian fashion SMEs
}

\author{
Romeo Bandinelli, Elisa d'Avolio, Rinaldo Rinaldi \\ Department of Industrial Engineering \\ University of Florence \\ Florence, Italy \\ romeo.bandinelli@unifi.it, elisa.davolio@unifi.it, rinaldo.rinaldi@unifi.it
}

\begin{abstract}
The dynamic and complex context in which Italian SMEs are competing has led to their choice to constitute collaborative networks, in order to improve their capabilities and to take advantage to more resources, such as skills and knowledge. The present study aims to assess the features of collaborative networks in the Italian companies working as suppliers in the fashion industry through a multiple case study research. A first literature review has shown several frameworks evaluating companies at a cross-industry level. Then, the Camarinha-Matos \& Afsarmanesh model has been chosen to assess the features of the networks analyzed. The Collaborative Networked Organizations (CNOs) in the Tuscan-Emilian districts appear qualitatively well developed but improvable in their structure and in their strategies' definition. Therefore, the present research describe the reality of CNOs in the Italian context, providing interesting insights for academicians, overviewing the main frameworks assessing CNOs, and also for managers that are facing the issues related to collaboration between SMEs.
\end{abstract}

Keywords - collaborative networks, CNO, SME, fashion industry

\section{INTRODUCTION}

Nowadays the enterprises are operating in a market characterized by high dynamism and turbulence, putting them in the condition to face more and more competitive challenges. Important phenomena, such as globalization, have had a massive impact on the relationship between customers and suppliers: the customer's needs are continuously updated and companies are experiencing new opportunities to grow and, on the other hand, also lots of criticalities. In this scenario, organizational flexibility, innovation and efficiency are becoming basic levers to increase companies' competitiveness.

Thus, in order to emerge in this complex, competitive and evolving environment, the enterprises are reorganizing their internal operations and their information technology systems. Companies are asked to revolutionize their entire modus operandi, through a reengineering of the manufacturing processes to reduce wastes and costs.

New collaborative networks of enterprises, i.e. Collaborative Networked Organizations (CNOs), may represent a solution to the emerging issues. Through their involvement in CNOs, companies may protect themselves from possible competitive risks and, contemporarily, they are able to extend their product and their attractiveness across a wider market.

In this context, collaborative networks show a high potential, not only in terms of the survival capability, but also for value creation through new capabilities to cope with innovation needs, uncertainty, mass customization, and fierce competition [1].

Moreover, a context characterized by a wide presence of Small and Medium Enterprises (SMEs) lends oneself to the decision to take part of a collaborative network. In fact, a SME is able to operate as part of a large organization when it works in a $\mathrm{CNO}$, continuing to behave as a single entity outside the $\mathrm{CNO}$ in order to achieve autonomy and flexibility.

In the Italian context a massive presence of SMEs is recordable. These companies descend from a strong handcrafted tradition that has brought them in the condition to be specialized in a single productive phase. The new CNOs allow SMEs to assimilate their knowledge in order to grow and innovate, not just trying to survive but to be really competitive in this global market.

According to a research carried out by Unioncamere in Italy, updated to 2011, the trend of the collaborative networks has sharply risen in 2011, reaching a number of 214 CNOs composed by 1065 SMEs. The same research also shows that about the $31 \%$ of the SMEs is located in the Tuscan-Emilian district.

This particular industrial district is composed by lots of SMEs: they are family-run companies that lend themselves often as first or second level suppliers to bigger enterprises. They belong to the fashion industry and are involved in the apparel, leatherwear and accessories sectors. Many SMEs work together in naturally and historically bounded areas with a potentially high impact on the environment.

This competitive potential of the district is severely limited because it lacks the ability to spontaneously aggregate its activities, a situation exacerbated by the absence of leader firms capable of providing direction for the system as a whole [2]. In such a scenario, SMEs in the center of Italy have perceived the need to expand their own business-borders and 
therefore sought the involvement of academia to drive the company strategy.

The authors have conducted a multiple case study research to examine whether or not the network configurations are different and whether or not each give the same priority to several issues analyzed in literature. Therefore, the purpose of the present study is to assess the cases of collaborative networked organizations in the accessories and leatherwear industries, through an investigation of the existing frameworks.

Firstly, the paper reviews the literature about collaborative networks, focusing on the role of sustainability and innovation. The characteristics of the main assessment frameworks for CNOs are then described. The employed methodology is presented in the third section, highlighting the research questions and the characteristics of the networks analyzed. The fourth section answers to the first research question, introducing the rationales that guided to the choice of a framework, assessing the decision about the more suitable one to the case study. Then, in order to answer to the second research question, data from questionnaires and interviews have been analyzed and the main result are summarized and discussed. The fifth section shows the conclusions, the implications of the study and its limitations, proposing also future research directions.

\section{LITERATURE REVIEW}

According to [3], a collaborative network is constituted by a variety of entities (e.g., organizations and people) that are largely autonomous, geographically distributed, and heterogeneous in terms of their: operating environment, culture, social capital, and goals. Nevertheless these entities collaborate to better achieve common or compatible goals.

The goal is to build a "collaborative advantage" in which multiple organizations together achieve something that individual organizations could not achieve alone [4]. Collaborative networks are seen as having an "organic" ability to adjust and reorganize their patterns and structures in order to improve their growth, sustainability and resilience.

In the past decades, firms have strived to achieve greater collaborative advantages with their supply chain partners [5]. The authors define a new kind of competitive advantage, the "collaborative advantage": it represents the strategic benefits gained over competitors in the marketplace through supply chain partnering and partner-enabled knowledge creation, and it relates to the desired synergistic outcome of collaborative activity that could not have been achieved by any firm acting alone.

Within their limited resources, SMEs must find ways to achieve production economies of scale, to market their products effectively, and to provide satisfactory support services. Reference [6] have noted that SMEs are flexible and more innovative in new areas, but can lack resources and capabilities. Large firms may be less flexible but will tend to have stronger resources to develop inventions into products or processes, and these resources act as complementary assets in attracting SMEs to collaborate with them.
The key objective of the collaborative strategy is to coordinate all the resources of the SME, suppliers, and contractors to best fulfil the demands of the existing customers and continuously expand the customer network [7].

Reference [8] propose an organizational approach to collaborative networks, underling the aspects for their existence and efficiency. The authors consider the importance of costs, competence sharing, resources, common goals, coordinators, sustainability and trust between the partners.

Early studies have identified the key drivers of success for example: effective support from senior management, a clear sense of mission and objectives, a strong leadership team with personal commitment, individual excellence of partners, importance to fits strategic goals of each partner, interdependence among partners and investment as tangible commitment of partners. On the other hand, the reasons behind the failures may be attributed to difficulties in participants' relationship, participants' dissatisfaction with outcome and/or organization structure of the collaboration [9].

Moreover, the literature on collaboration identifies some motives and benefits associated with manufacturing interenterprise collaboration [10]: to increase the market share, asset utilization, to enhance customer service, to reduce the cost and time of product development, to share costs, to increase the quality of the products, to increase/enhance skills and knowledge, to have a technological gain as participating firm, to achieve economies of scale in production, to decrease risk of failure of product development, to reduce inventory in the face of increasing technological complexity and rapid rate product development and obsolescence, to gain rapid access to markets, to increase flexibility, to attain international presence for SMEs, to cope with changing dynamics, to have an effective knowledge management, to have access to resources (e.g., skills, knowledge) that are usually well beyond those of a single player, to learn from other participants in the network.

The literature review step has shown that the majority of the papers analyzed is composed of case studies and empirical researches. The first ones aim to describe the features of collaborative networks existing in a particular industry and the second ones, analyzed in an appropriate sub-section, describe the frameworks or the modelling intent to evaluate collaborative networks.

In particular, reference [4] examine a case study of an employment-services non-profit network, while [2] present the case of a CNO in a particular district in Italy, characterized by a high technical-productive specialization in the mechanical and printing field.

Moreover cases conducted in the fashion industry exist. Reference [11] describe the evolution of the French apparel industry from district to network forms, underlining the resemblance and the differences to Italian garment regions, such as Emilia-Romagna and Veneto. Reference [12] investigate the supply networks of different types of retailers in Sri Lanka, also assessing the criteria that SMEs have to respect in order to participate in global supply networks. Reference [13] propose an innovative methodological approach and ICT platform to support non-hierarchical collaboration between 
SMEs: the authors adopted the case study methodology in two business realities, belonging to the fashion industry, in Portugal and Italy. Thanks to the support of their model, communication and information shared by all partners appear more streamlined, ensuring transparency and creating a truthful collaboration environment.

\section{A. Sustainability and innovation in supply networks}

An important issue, which has been stressed by several authors, is the environmental approach to collaborative networks, meant as their relationship with sustainability.

As the future challenge is to develop a sustainable global economy, it becomes increasingly important for firms to evaluate the impact that a sustainable supply chain strategy has also on environmental aspects. A sustainable organization contributes to sustainable development by simultaneously delivering economic, social and environmental benefits, or what has been termed "the triple bottom line" or 3BL [14].

According to [15] the complexity of environmental issues requires that firms embracing environmental sustainability into their strategies and activities collaborate with a wide range of external parties and include a broad range of stakeholders that can be a source of environmental knowledge and competencies outside the firm's main domain. Collaborations are important for companies to face environmental challenges, as in most cases they are inherently complex, thereby requiring the integration of different types of knowledge and competencies, which are likely to come from diverse organizations and/or industries. The authors empirically test the effect of collaborations on environmental performance, by simultaneously considering environmental collaborations with different types of actor

Moreover, reference [16] explore holistic and systemic strategies for seeking integrated solutions at both intra- and inter-enterprise levels for lowering resources input, enhancing resources productivity, reducing wastes and emissions, and lowering operating costs within an enterprise and between industrial networks based-on sustainable Collaborative Networked Organizations models. They introduce the concept of Green Virtual Enterprise Breeding Environment (GVBE), as a long-term strategic alliance of green enterprises and their related support institutions aimed at offering the necessary conditions to efficiently promote the sharing and recycling of resources.

An important contribution to the assessment of sustainable practice in supply networks is represented by [12]. The authors adopt the three pillars of 3BL as the dimensions of their assessment about sustainability practices of two international clothing supply networks.

Another issue that has emerged during the literature review phase, has been the role of innovation. It is agreed that, while SMEs' flexibility and specificity can be advantages in accelerating innovation, few of them have sufficient capacity to manage the whole innovation process by themselves, and this encourages them to collaborate with other firms. Reference [6] focus on open innovation strategies in SMEs, suggesting a network model that emphasizes the role of intermediaries in linking SMEs.

Reference [17] analyze theoretically and empirically the role of different types of collaborative networks in the achievement of product innovations and their degree of novelty. They found that implementation of additional capabilities from outside has a positive effect on innovation achievement, noticing the positive relationship between collaboration and product innovations.

Reference [18] examines the effects of different types of partners on product innovation performance. His framework indicates that the product innovation performance of a firm is affected by its collaborative networks in terms of different types of partners.

\section{B. Collaborative network classifications and assessment frameworks}

One of the most important contributions to the classification of collaborative networks models is represented by [3]. They list the recent manifestations or variants of CNs [19]: Virtual Enterprises (VE), Virtual Organizations (VO), Dynamic Virtual Organizations (DVO), Extended Enterprises (EE) and Virtual Teams (VT). These variants have been studied by several authors, who have also investigated models, framework and other empirical findings [20], [21].

Another classification concerns the vertical, horizontal and spatial complexity of supply networks [12]. Vertical complexity is assessed in terms of the number of tiers in the network, horizontal complexity in terms of the number of different entities in the same tier and spatial complexity in terms of the geographical dispersion of entities in the supply network. Moreover, the Virtual Development Office (VDO) is specifically devoted to promote new and innovative business opportunities within the $\mathrm{CNO}$.

For the aim of the present study, the main works concerning the evaluation of the CNOs, in their more general meaning, have been analyzed. In the period that ranges from 2004 to 2012, several authors have adopted different approaches that will be described in detail.

Reference [22] have proposed a framework capable to support the estimation of the potential performance for a network of firms, by identifying some of the main factors that must be considered to build a set of synthetic KPI. Starting from some attribute measures at a single firm level (ICT skills and resources, knowledge and competencies, cost per hour, performance rating, partner availability, level of commitment and level of risk), also the definition of team performance indicators (technological integration, knowledge sharing attitude, coordination skills, network robustness) has been proposed. Firstly, single firms factors must be identified and evaluated, then, those factors can be elaborated and linked to enable the evaluation of the relationships and the interactions among the partners in the whole potential network.

The Supply Chain Management (SCM) Maturity Model (MM) developed by [23] is characterized by five stages of maturity and the decision areas that are based on the SCOR Model. The SCOR framework has been chosen to 
conceptualize the supply chain management maturity model, owing to its process orientation and wide adoption by the supply chain academic and practitioner communities. The five stages of maturity show the progression of activities toward effective SCM and process maturity. An organization must evolve through these levels to establish a culture of process excellence.

Reference [24] introduce ICoNOs MM in order to facilitate business-IT alignment in networked organizations. In collaborative networked organizations business-IT alignment (B-ITa) is a key issue that requires continuous attention. Improving IT-business alignment is possible in order to achieve a greater integration in the $\mathrm{CNO}$ and a more efficient use of IT. The Model is composed of four domains: partnering structure, IS architecture, process architecture and coordination. In each of the domains, also process areas are present. Process areas are sets of activities that are performed to improve a particular domain. The ICoNOs MM has five levels of maturity (incomplete, isolate, standardized, quantitatively managed and optimized) that are used to describe an improvement for a CNO that wants to enhance processes to achieve B-ITa. To reach a particular level, a CNO must satisfy all the set of process areas that are targeted for improvement in a particular B-ITa domain.

Moreover, reference [25] propose a comprehensive modelling framework as a first step towards the elaboration of a reference model for collaborative networks. The Framework identifies four dimensions from an endogenous perspective of collaborative networked organizations: structural, componential, functional, and behavioral. Each of these dimensions is characterized by a set of sub-dimensions that delineate the profile. The structural dimension represents the CNO structure and its composition is described by two subdimensions, named actors/relationships and roles. The componential dimension describes all types of resources: hardware/software resources, human resources and information/knowledge resources. The functional dimension concerns processes and procedures and it includes: processes, auxiliary processes and methodologies. The behavioral dimension expresses principles, rules and governance policies that constrain and guide the behavior of the $\mathrm{CNO}$ and its members over time.

In parallel with the endogenous perspective there is an exogenous perspective, which assesses the relationship of the network with external actors. The exogenous prospective consists of four dimensions too: market, support, societal and constituency dimension. The market dimension expresses the relationships with customers and competitors. The support dimension describes all problems related to the services provided by third parties: certification insurance and training services. The societal dimension captures the issues related to the interactions between the $\mathrm{CNO}$ and the society in general. The constituency dimension focuses on the interactions to the universe of potential new members of the CNO.

Another important contribution to the assessment of collaborative networks is based on the study proposed by [12]: they use case studies evidence to evaluate the network configurations and supply chain practices of the two types of retailers, highlighting the sustainability issues. In particular, The dimensions identified are based on the three pillars of 3BL: environmental (a stable base of resources, ecosystems and biodiversity, fresh water, recycling, low carbon), economic (efficiency, jobs and wealth creation, producing goods and services, prosperity), social (social services, inclusion, equity, accountability, culture, groups, places and participation). To each of these dimensions correspond several assessing criteria characterizing the supply network: stability, quality assurance processes, workflow rights, social projects and environmentaloriented strategy. Understanding the structure, operation and sustainability agendas of different retailers is important for SMEs in understanding their abilities to participate in global supply networks.

\section{METHODOLOGY}

The present study aims to assess the features of collaborative networks in the Italian fashion industries through a multiple case study research. A first literature review has shown several frameworks evaluating companies at a crossindustry level focusing on different key-points. The particular industrial district of Tuscany is packed with SMEs manufacturing leather goods and accessories, which are recognizing the importance to build collaborative business networks in order to reach specific business goals.

In this context, a case study analysis has been performed in order to validate the frameworks gathered from literature and to understand to what extent Italian SMEs are able to adopt a collaborative behavior in the network built. In particular, two main research questions (RQs) emerge:

RQ1: which of the frameworks proposed in literature matches the need to assess the Italian collaborative networks?

RQ2: what about the maturity of the CNOs interviewed during the case study?

A multiple case study has been designed to answer the RQs. The case study is a research strategy which focuses on understanding the dynamics present within single settings. According to [26] a number between 4 and 10 cases usually works well in these researches, so the considered sample is sufficient to give an accurate account in an empirical research.

A questionnaire has been used in order to support the interviews conducted. It is composed of two main parts. The first one is devoted to describe the general features of the enterprise: its goal is to introduce the companies involved in the network and to identify the industrial background. Therefore, this first part contains open questions about the interviewee's role, the company's size, organogram and core business, the main competitors and future direction of investment.

The second part aims to evaluate the characteristics of the network. While the first one has been distributed to each network's partner, this second part has been given just to the network manager or to the authority aware of the entire CNO and its inner working. This questionnaire's section is composed of closed questions and it is structured according to the 
dimensions identified by the chosen framework, assessing the CNO maturity.

The direct contact to network managers and other authorities belonging to the CNOs allowed us to carry out structured interviews, often backed up by unstructured interviews and interactions. Multiple methods such as questionnaires, direct observations and content analysis of document has been used to perform data triangulation. The latter, through the employment of different methods of data collection, is able to strengthen the validity of the research [27]. Data collected have been recorded and then analyzed.

The interviews were carried out basing on a questionnaire composed of two main parts: a company presentation and an assessment of the $\mathrm{CNO}$, previously chosen on the literature review basis. The latter is the core section that aims to evaluate each dimension of the model adopted.

In order to become familiar to each case as a standalone entity a primary within case analysis has been performed. Then a comparison between the cases has been conducted and a cross-case analysis has been carried out. The multiple case study research has involved a sample of four companies: its main features have been represented in Table 1. Basically the companies interviewed sell leatherwear and accessories products in the province of Florence and represent businesses with a high artisanal mark. Each collaborative network is composed of a relatively small number of key-players (one or two): the first case adopts a unique form of aggregation that is the only one visible to the customer while, in the other cases, two companies are allowed to accept orders from the customer. A variable number of other partners and subcontractors, which ranges from 3 to 15 , is also present. Coming to the network structure, the first case study is characterized by a vertical one: three key players collaborate to cover the overall processes required to manufacture metallic accessories (polishing, galvanic and mechanical office). The other three cases are hybrids because key players are involved in the same process, while in their relationship with the other partners they present a vertical structure.

Table 1 - Sample

\begin{tabular}{|c|c|c|c|c|c|}
\hline $\begin{array}{c}\text { Case } \\
\text { study }\end{array}$ & $\begin{array}{c}\text { Interviewee's } \\
\text { role }\end{array}$ & $\begin{array}{c}\text { Main } \\
\text { product }\end{array}$ & $\begin{array}{c}\mathbf{N}^{\circ} \text { of } \\
\text { key } \\
\text { players }\end{array}$ & $\begin{array}{c}\mathbf{N}^{\circ} \text { of } \\
\text { other } \\
\text { partners }\end{array}$ & $\begin{array}{c}\text { Network } \\
\text { structure }\end{array}$ \\
\hline 1 & $\begin{array}{c}\text { CNO } \\
\text { authority }\end{array}$ & $\begin{array}{c}\text { Metal } \\
\text { accessories }\end{array}$ & 1 & 3 & Vertical \\
\hline 2 & $\begin{array}{c}\text { CNO } \\
\text { manager }\end{array}$ & Leatherwear & 2 & 15 & $\begin{array}{c}\text { Horizontal } \\
\text { between } \\
\text { key } \\
\text { players. } \\
\text { Vertical } \\
\text { between } \\
\text { key-players } \\
\text { and other } \\
\text { partners }\end{array}$ \\
\hline 3 & $\begin{array}{c}\text { CNO } \\
\text { manager }\end{array}$ & Leatherwear & 2 & 6 & $\begin{array}{c}\text { Horizontal } \\
\text { between } \\
\text { key } \\
\text { players. }\end{array}$ \\
\hline
\end{tabular}

\begin{tabular}{|c|c|c|c|c|c|}
\hline & & & & $\begin{array}{c}\text { Vertical } \\
\text { between } \\
\text { key-players } \\
\text { and other } \\
\text { partners }\end{array}$ \\
\hline 4 & $\begin{array}{c}\text { CNO } \\
\text { manager }\end{array}$ & Leatherwear & 2 & 12 & $\begin{array}{c}\text { Horizontal } \\
\text { between } \\
\text { key } \\
\text { players. } \\
\text { Vertical } \\
\text { between } \\
\text { key-players } \\
\text { and other } \\
\text { partners }\end{array}$ \\
\hline
\end{tabular}

During the interviews, qualitative and quantitative evidences have been combined: the qualitative data are useful in the understanding the rationale of theory, underlying relationships revealed in the quantitative data, or may suggest directly theories which can then be strengthened by quantitative support [26].

\section{CASE STUDY RESULTS}

The wide presence of SMEs in the Italian context has encouraged an in depth-analysis evaluating the maturity of the collaborative networks. The increasing globalization and competitiveness have triggered the SMEs to find in the CNOs the way to preserve their core characteristics and to be reactive in the actual complex market.

As a first evidence, is it possible to observe that the SMEs in the analyzed context have not implemented a Performance Measurement System (PMS) to evaluate operational and strategic behaviors, because of a lack in culture, instruments and tools or because they decided to give more prominence to other tactical issues.

Despite this fact, the models proposed in the literature and described above are able to support companies in conducting a qualitative evaluation of their collaborative networks. For this reason, in this section the answers to the RQs have been provided.

\section{A. The choice of a more suitable framework to the sample considered}

The goal of this section is to answer to the first RQ, concerning the choice of the framework proposed in literature that matches the need to assess the Italian collaborative networks.

The closing contact with the collaborative network managers, during the case study research, has allowed identifying the characteristics that are required to assess the maturity of CNOs.

In Table 2 a classification of the main models' features has been provided. First of all, a framework has to be processoriented, in order to cover all the business processes carried out by the companies interviewed. Moreover, the framework may adopt a static or a dynamic approach: the latter allows to better 
understand the evolution of a company's processes during its lifecycle. Then, each model may be conceived to cover the processes of the SC or of the entire collaborative network: the aim of this study is basically devoted to CNOs. Among the possible classifications of business processes, the Porter's value chain has been chosen [28]: primary activities, (operations, inbound and outbound logistics, marketing and sales and service) and support ones (firm infrastructure, Human resource management, technology and procurement) have been taken into account. The recent and increasingly debated issue of sustainability has been added to the list of support activities in order to consider also the environmental aspects at a network level.

The model proposed by Raffa [22] is not process-oriented and it is also static and quantitative because discusses several performance indicators, omitting the developments of the business with time. It concerns the overall $\mathrm{CNO}$, but the evaluation factors identified are able to cover just the support activities, excluding the theme of sustainability. The SCM MM [23], as all the other models, describes a process-oriented and dynamic framework. It, unlike the others is specifically devoted to describe $\mathrm{SC}$ process, rather that $\mathrm{CNO}$ ones. The use of the SCOR, ensures the coverage of the primary activities, but the support ones have not been taken into consideration. Moreover, The ICoNOs MM [24] omits the majority of the primary activities.

Therefore two main models stand out for their processoriented approach and their concern to network activities: the MacCarthy and Jayarathne [12] model and the CamarinhaMatos \& Afsarmanesh [25] one. Both adopt a qualitative approach, highlighting also the importance of sustainability issues. Even if a trade-off between quantitative and qualitative methodologies is recommended, in this particular case the qualitative one stands out.

For the aim of the present study, the eight dimensions proposed by the Camarinha-Matos \& Afsarmanesh [25] have been chosen as the most representative for qualitative modeling collaborative networks composing the sample. The questionnaire, that has been distributed to the companies interviewed, contained in its second section the chosen model features.

Table 2 - Model's features

\begin{tabular}{|c|c|c|c|c|c|c|c|c|c|c|c|c|}
\hline & \multicolumn{3}{|c|}{ Tendency } & \multicolumn{4}{|c|}{ Primary activities } & \multicolumn{5}{|c|}{ Support activities } \\
\hline Framework & $\begin{array}{l}\text { Process- } \\
\text { oriented }\end{array}$ & $\begin{array}{l}\text { Static/Dynami } \\
\text { c }\end{array}$ & $\begin{array}{l}\text { Process } \\
\text { coverage: } \\
\text { SC/CNO }\end{array}$ & Ope. & Log. & $\begin{array}{l}\text { M\& } \\
\text { S }\end{array}$ & Ser. & F.In. & $\begin{array}{l}\text { HR } \\
\text { m. }\end{array}$ & Tec. & Pro. & Sus. \\
\hline Raffa [22] & No & Static & $\mathrm{CNO}$ & & & & & $\mathrm{X}$ & $\mathrm{X}$ & $X$ & $\mathrm{X}$ & \\
\hline $\begin{array}{l}\text { Lockamy III and } \\
\text { McCormack [23] }\end{array}$ & Yes & Dynamic & SC & $\mathrm{X}$ & $\bar{X}$ & $\mathrm{X}$ & $\mathrm{X}$ & & & & & \\
\hline Tapia et al., [24] & Yes & Dynamic & $\mathrm{CNO}$ & $\mathrm{X}$ & & & & $\mathrm{X}$ & $\mathrm{X}$ & $\mathrm{X}$ & $\mathrm{X}$ & \\
\hline $\begin{array}{l}\text { Camarinha-Matos } \\
\& \quad \text { Afsarmanesh } \\
{[25]}\end{array}$ & Yes & Dynamic & $\mathrm{CNO}$ & $\mathrm{X}$ & $\mathrm{X}$ & $\mathrm{X}$ & $\mathrm{X}$ & $\mathrm{X}$ & $\mathrm{X}$ & $\mathrm{X}$ & $\mathrm{X}$ & $\mathrm{X}$ \\
\hline $\begin{array}{l}\text { MacCarthy and } \\
\text { Jayarathne [12 }\end{array}$ & Yes & Dynamic & $\mathrm{CNO}$ & $\mathrm{X}$ & $X$ & & & $\mathrm{X}$ & $\mathrm{X}$ & & $\mathrm{X}$ & $\mathrm{X}$ \\
\hline
\end{tabular}

Key: Ope.:Operations;Log.=Inbound/Otbound Logistics; M\&S=Market \& Sales; Ser.:Service; F.In.:Firm Infrastructure; HR m.: Human Resource management; Tec.:Technology; Pro.: Procurement; Sus.:Sustainability

\section{B. Eight dimensions to evaluate CNOs' maturity}

After the questionnaire administration, the outcomes have been deeply analyzed in order to answer to the second RQ, concerning the maturity of the CNOs interviewed.

During the analysis phase, to each dimension examined, basing on the answers, a score has been assigned. Given the lack of many case studies and associated data, the analysis has been mainly qualitative.

The first case represents one of the first CNO born in Italy in the metal accessories industry. It's a network that has developed an acceptable level of maturity and in particular, the exogenous dimensions are more developed than the endogenous ones.

From an endogenous perspective, the Structural dimension is not yet really mature, because the network does not show a business model and an integrated management approach, even if a network management authority exists. The Componential dimension is supported by continuous exchange of experiences, also by informal meetings. Both human and knowledge resources have been identified. The partners have implemented Information Technology Systems (ITSs), but they are not integrated because specific interface modules still miss. The 
Functional dimension is not developed: processes and procedures have not been formalized and a set of Key Performance Indicators (KPIs) has not been individuated. The Behavioral dimension is characterized by well-defined constraints, conditions and agreements: the presence of a network management authority ensures the compliance to the rules identified by the partners.

From the exogenous perspective, the market dimension is developed in terms of marketing and branding but a clear market strategy has not been defined and also the positioning in the competitive context has not been assessed. The Support dimension is the most developed within the first case because the external coaching and the certification services are ensured by consultants with whom the companies have established a relationship of mutual confidence. The Societal dimension is well developed because of the importance the enterprises attribute to the Corporate Social Responsibility (CSR) and to the sustainability issues. The Constituency dimension is a critical part of the network assessment: the companies have not evaluated the opportunity to include other partners in the CNO. They precise that, whenever other members will take part to the community, the roles of management and control will belong to the founders. This aspect demonstrates the need to limit the rules of adhesion and it makes the network less dynamic: the partners prefer not to look for the best suppliers for a given manufacturing process, containing the market revenue policies. In the $\mathrm{CNO}$ context, instead, the key players should be interested to find the best performers, excluding the partners that are not able to collect orders, decreasing the market share.

The second, the third and the fourth case studies, belonging to the leatherwear industry, represent three networks very similar in terms of maturity. In these cases the endogenous perspective is more developed than the exogenous one, but both result more mature if compared to the first case.

The Structural dimension is characterized by roles and responsibility identified a priori. The actors and their relationship are also well-defined. In the Componential dimension the use of job description and the importance of training contribute to a mature human resources management, but the ITSs are not present and obviously not integrated to each other. The Functional dimension includes the definition of processes, through flow charts, and the need for KPIs measuring the process performances. The Behavioral dimension appears really mature due to the rules and conditions which are frequently controlled, ensuring accuracy and fairness.

Within the exogenous perspective, the Market dimension is the less developed because of the peculiarities of accessories industry. In fact, the companies do not establish their competitive advantage on a wide range of products, because the most important aspect in their market strategy is the enhancement of quality in products and in processes. Nevertheless, the networks are able to evaluate their market positioning and to identify their main competitors. The Support dimension and the Societal dimension are really mature, as in the first case: in particular an increasing focus on processes reviewing and on use of raw material with low level of environmental impact is reported. Referring to Constituency dimension, the networks show a major openness to new possible entrants, but define constraints and rules that they have to respect.

The majority of the SMEs interviewed could not have survived without relationships to bigger enterprises. This way, they have reached a modernization of manufacturing processes and the access to skills, resources and assets to expand their market share. On the other hand, the bigger companies have achieved superior levels of quality and specialization. Both bigger and smaller enterprises can take advantage from their capability to manage the entire set of processes required to realize a finished product. This situation enables them to gain access to the international market, increasing the network visibility.

Nowadays the structural consolidation of Italian companies through aggregation processes is more than ever important to face the foreign competition. The phenomenon of collaborative networks, in fact, has strengthened the market dimension of the SMEs and their competitive position, exploiting and feeding different synergies and increasing efficiency and effectiveness in the entire value chain.

However, the actual conditions of CNOs in the district analyzed may be improved leveraging on different issues, such as the definition of a VDO, as defined in literature, embodying the authority that promotes business opportunities and coordinates the entire CNO. This will enhance the structural dimension and also will impact on societal and constituency ones, defining policies and long-term strategies. Moreover, the network dynamism may be improved through the identification of skilled partners, forsaking the closing and obsolete approach that gives bargaining power to the network founders. A specific training should be provided to prepare the SMEs to the collaborative approach, clarifying the benefits achievable through CNOs and the required efforts.

The companies interviewed have reported, as the main motivations to constitute a collaborative network, the need to improve the innovation level, to manufacture a finished product, exploiting different skills and also the opportunity to enhance their competitiveness. The criticalities that these SMEs have had to face are related to the difficulties in coordinating different enterprises and in sharing know-how.

\section{CONCLUSIONS AND FURTHER DEVELOPMENTS}

The Tuscan-Emilian industrial district is constituted by lots of SMEs, specialized in particular manufacturing processes that have recently decided to collaborate and to constitute Collaborative Networked Organizations. In this context, the authors have performed a case study research aiming to assess the features of collaborative networks in the metal accessories and leatherwear industries.

The first step within the research has been the identification and the analysis of the main literature concerning CNOs and framework evaluating their level of maturity. This phase has allowed, from a methodological viewpoint, the authors to answer the first research question about the need to assess the Italian collaborative networks through frameworks proposed in 
literature. The Camarinha-Matos \& Afsarmanesh [25] model has been chosen because of its capability to cover the network processes and to assess their maturity.

Then, a questionnaire has been designed, evaluating the dimensions described in the model for the networks included in the case study research. The questionnaire has been distributed and the outcomes have been deeply analyzed, with the aim to answer to the second research question about the maturity of the CNOs interviewed.

The analysis has separated the accessories and leatherwear industries because they are internally homogeneous and differ for many aspects. Endogenous and exogenous dimension have been discussed highlighting the weakness and the strength of the cases collected. The major lacks have been reported in the Structural, Functional and Constituency dimensions for the metal accessories industry. The leatherwear industry, instead, appears more mature in the overall dimensions even if the Information resources appear not-well managed.

In conclusion, the CNOs in the Tuscan-Emilian district appear well developed but, for sure, improvable in their structure and in their strategies' definition.

The model is appropriated to qualitatively assess the overall characteristics of the context analyzed, but a quantitative approach is required in order to evaluate the operations of the entire CNO. Therefore, future research directions may be addressed to the development of a framework containing qualitative and quantitative variables to better assess CNOs. The direct contact with network managers and the analysis of more than four case studies will support the comprehension of collaborative networks and their features.

In spite of the limits of the present research, basically due to the low number of cases analyzed, it immortalizes the reality of CNOs in the Italian context. Moreover it provides interesting insights for academicians, overviewing the main frameworks assessing CNOs, and also for managers that are facing the issues related to collaboration between SMEs.

\section{REFERENCES}

[1] L. M. Camarinha-Matos, "Collaborative networked organizations: Status and trends in manufacturing", Annual Reviews in Control, vol. 33, 2009, pp. 199-208.

[2] S. Saetta, L. Tiacci, L. Cagnazzo, "The innovative model of the VDO for collaborative networked enterprises: the GPT network case study", International Journal of Computer Integrated Manufacturing, vol. 26, 2013, pp.1-13.

[3] L. M. Camarinha-Matos, H. Afsarmanesh, "Collaborative networks: a new scientific discpline", Journal of Intelligent Manufacturing, vol. 16, 2005, pp. 439-452.

[4] E. Baker, M. Kan, "Developing a collaborative network organization: leadership challenges at multiple levels", Journal of Organizational Change Management, vol. 24, 2011, pp. 853-875.

[5] M. Cao, Q. Zhang, "Supply chain collaborative advantage: a firm's perspective", Int. Journal of Production Economics, vol. 128, 2010, pp. 358-367.

[6] S. Lee, G. Park, B. Yoon, J. Park, "Open innovation in SMEs-An intermediated network model”, Research Policy, vol. 39, 2010, pp. 290300 .
[7] H. W. Lin, S. V.Nagalingam, S. S. Kuik, T. Murata, "Design of a Global Decision Support System for a manufacturing SME: Towards participating in Collaborative Manufacturing", International Journal of Production Economics, vol. 136, 2012, pp.1-12.

[8] J. Eschenbächer, N. Zarvic, "Towards the explanation of goal-oriented and opportunity-based networks of organizations", Journal of Manufacturing Technology Management, vol. 23, 2012, pp. 1071-1089.

[9] J. Parung, U. S. Bititci, "A metric for collaborative networks", Business Process Management Journal, vol. 14, 2008, pp. 654-674.

[10] C.-M. Chituc, C. Toscano, A. Azevedo, "Interoperability in Collaborative Networks: Independent and industry-specific initiatives The case of the footwear industry", Computers in Industry, vol. 59, 2008, pp. 741-757.

[11] B. Courault, P. B. Doeringer, "From hierarchical districts to collaborative networks: the transformation of the French apparel industry", Socio-Economic Review, vol. 6, 2008, pp. 261-282.

[12] B.L. MacCarthy, P.G.S.A. Jayarathne, "Sustainable collaborative supply networks in the international clothing industry: a comparative analysis of two retailers", Production Planning \& Control: The Management of Operations, vol. 23, 2012, pp. 252-268.

[13] A. Shamsuzzoha, T. Kankaanpaa, L. Maia Carneiro , R. Almeida, A. Chiodi, R. Fornasiero, "Dynamic and collaborative business networks in the fashion industry", International Journal of Computer Integrated Manufacturing, vol. 26, 2013, 125-139.

[14] M. J. Markley, L. Davis, "Exploring future competitive advantage through sustainable supply chains", International Journal of Physical Distribution \& Logistics Management, vol. 37, 2007, pp. 763-774.

[15] V. Albino, R. M. Dangelico, P. Pontrandolfo, "Do inter-organizational collaborations enhance a firm's environmental performance? a study of the largest U.S. companies", Journal of Cleaner Production, vol. 37, 2012, pp. 304-315.

[16] D. Romero, A. Molina, "Green Virtual Enterprise Breeding Environments: A Sustainable Industrial Development Model for a Circular Economy", Collaborative Networks in the Internet of Services, IFIP Advances in Information and Communication Technology, vol. 380, 2012, pp. 427-436.

[17] M. J. Nieto, L. Santamarìa, "The importance of diverse collaborative networks for the novelty of product innovation", Technovation, vol. 27, 2007, pp. 367-377.

[18] K. H. Tsai, "Collaborative networks and product innovation performance: Toward a contingency perspective", Research Policy, vol. 38, 2009, pp. 765-778.

[19] L. M. Camarinha-Matos, H. Afsarmanesh, M. Ollus, "Ecolead: a holistic approach to creation and management of Dynamic Virtual Organizations", Collaborative Networks and Their Breeding Environments, IFIP - The International Federation for Information Processing, vol. 186, 2005, pp 3-16.

[20] E. Esposito, P. Evangelista, "Investigating virtual enterprise models: literature review and empirical findings", Interantional Journal of Production Economics, in press.

[21] B. Ezzeddine, B. Abdellatif, B. Mounir, "An intelligent Framework for the Cooperation in the Extended Enterprise Environment", 2011 4th International Conference on Logistics (LOGISTIQUA), pp. 379 - 384.

[22] L. Raffa, P. Rippa, A. Sgalambro, "A methodological approach for the evaluation of an Extended Enterprise: a two-tier framework based on key performance indicators”, MITIP 2007, 6-7 September, Florence.

[23] A. Lockamy III, K. McCormack, "The development of a supply chain management process maturity model using the concepts of business process orientation", Supply Chain Management: An International Journal, vol. 9, 2004, pp. 272-278.

[24] R. S. Tapia, "ICoNOs MM: The IT-Enabled Collaborative Networked Organizations Maturity Model", Leveraging Knowledge for Innovation in Collaborative Networks, IFIP Advances in Information and Communication Technology, vol. 307, 2009, pp 591-599.

[25] L. M. Camarinha-Matos, H. Afsarmanesh, "A comprehensive modelling framework for collaborative networked organizations", Journal of Intelligent Manufacturing, vol. 18, 2007, pp. 529-542. 
[26] K. M. Eisenhardt, "Building theories from case study research", The Academy of Management Review, vol. 14, 1989, pp. 532-550.

[27] C. Voss, N. Tsikriktsis, M. Frohlich, "Case research in operation management", International Journal of Operations \& Production Management, vol. 22, 2002, pp. 195 - 219.
[28] M. E. Porter, Competitive Advantage: Creating and Sustaining Superior Performance, Simon and Schuster, 2008. 